\title{
Growth performance, intestinal microflora, plasma fatty acid profile in broiler chickens fed herbal plant (Euphorbia hirta) and mix of acidifiers.
}

\begin{abstract}
The aim of the current study was to evaluate two feed additives containing Euphorbia hirta and acidifiers on broiler chickens performance, gut microflora and blood fatty acid profiles. A total of 240 one-day-old male broiler chicks were randomly assigned in groups of 15-16 floor pens. On day one, the chicks were assigned to one of 4 dietary treatments; (1) basal diet (control), (2) basal diet. +. 7.5. g/kg E. hirta (EH7.5), (3) basal diet. +. 1.5. g/kg acidifier (OA) and (4) basal diet. +. 7.5. g/kg E. hirta+. 1.5. g/kg acidifier (EHOA). Body weight and feed intake were measured weekly and feed conversion ratio (FCR) was calculated. On days 21 and 42 , eight birds from each dietary treatment were chosen at random for ileum viable bacterial counts. On day 42, thiobarbituric acid reactive species as an indicator of lipid peroxidation level were measured. The EH7.5, EHOA and OA supplementation improved overall FCR $(\mathrm{P}=0.0275)$. The serum of chickens fed with EH7.5, OA and EHOA showed the lower malondialdehyde value than that of chickens fed the control diet $(\mathrm{P}=0.0068)$. On day 42, the lowest total bacteria count and Escherichia coli was observed in EH7.5, OA and EHOA fed birds $(\mathrm{P}<0.0001)$. In conclusion, the results of the present study suggest that dietary supplementation with $\mathrm{E}$. hirta in combination with acidifier have a positive impact on FCR and their combination displays antioxidant and antibacterial characteristics in broiler chickens.
\end{abstract}

Keyword: Acidifier; Antibacterial; Antioxidant; Broiler; Euphorbia hirta; Herbal plant 